\title{
A NEW BITOPOLOGICAL PARACOMPACTNESS
}

\author{
T. G. RAGHAVAN and I. L. REILLY \\ (Received 12 January 1984; revised 4 September 1985) \\ Communicated by J. H. Rubinstein
}

\begin{abstract}
In this paper we define a new generalization of paracompactness for bitopological spaces. $\left(X, \tau_{1}, \tau_{2}\right)$ is $\delta$-pairwise paracompact if and only if every $\tau_{i}$ open cover admits a $\tau_{1} \vee \tau_{2}$ open refinement which is $\tau_{1} \vee \tau_{2}$ locally finite. Every quasimetric space $\left(X, \tau_{p}, \tau_{q}\right)$ is $\delta$-pairwise paracompact. An analogue of Michael's characterization of regular paracompact spaces is proved for $\delta$-pairwise paracompact spaces.

1980 Mathematics subject classification (Amer. Math. Soc.): 54 D 18, 54 E 55.
\end{abstract}

\section{Introduction}

The study of bitopological spaces was initiated by Kelly [5] when he investigated a nonsymmetric generalization of metric spaces, namely, the quasimetric spaces of Wilson [10]. Every quasimetric led to the consideration of a conjugate quasimetric and to the study of the structure of bitopological spaces. Since then, considerable effort has been expended in obtaining appropriate generalizations of standard topological properties in the bitopological category. This process of generalization met with considerable difficulty with the covering properties. The basic problem is the stability of bitopological spaces satisfying some covering axiom in the presence of the pairwise Hausdorff separation property, when the two topologies on the space may collapse and revert to the unitopological setting. With particular reference to bitopological compactness, some of the problems, and various definitions and their scope, have been discussed by Cooke and Reilly [1].

The second author acknowledges a grant from the University of Auckland Research Fund. (C) 1986 Australian Mathematical Society 0263-6115/86\$A2.00+0.00 
Paracompactness in the bitopological setting appears to be the most intractable of all. In this paper, we introduce and study a new notion of pairwise paracompactness. Our main result is that our new notion allows for the first time a bitopological version of Michael's classical characterization of regular paracompact spaces. Let us write the abbreviation b.t.s. for bitopological space and $n b d$ for neighbourhood. If $\tau_{i}$ is a topology for the set $X$, and if $A \subset X$, we write $\tau_{i} \mathrm{cl} A$ for the closure of $A$ taken in the topology $\tau_{i}, \tau_{i}\langle x\rangle$ for the set of all $\tau_{i}$ open nbds of $x$, and $\tau_{i} \mid A$ for the relative topology of $\tau_{i}$ on $A$.

\section{Some initial results}

Let $(X, \tau)$ be a topological space. Let $\mathscr{U}$ be an open cover of $X$. Any open cover $\mathscr{V}$ (of $X$ ) is called a refinement of $\mathscr{U}$ if every $V \in \mathscr{V}$ is a subset of some $U \in \mathscr{U}$. Further, $\mathscr{V}$ is called $\mathscr{G}$ locally finite if every point $x(\in X)$ belongs to a set $G \in \mathscr{G}$ such that $G$ meets only finitely many members of $\mathscr{V}$. It should be noted that $\mathscr{G}$ is a cover of $X$. We say $X$ is $\tau$ paracompact with respect to $\mathscr{G}$ if every $\tau$ open cover of $X$ admits a $\tau$ open refinement which is $\mathscr{G}$ locally finite. Obviously this leads to our definition [6, Definition 2] of $\tau_{1}$ paracompact with respect to $\tau_{2}$ for a b.t.s. $\left(X, \tau_{1}, \tau_{2}\right)$. Let us call $\left(X, \tau_{1}, \tau_{2}\right) R R$-pairwise paracompact if $X$ is $\tau_{i}$ paracompact with respect to $\tau_{j},(i, j=1,2 ; i \neq j)$. Another definition of pairwise paracompactness which appeared early was given by Fletcher, Hoyle III and Patty [3]. A b.t.s. $\left(X, \tau_{1}, \tau_{2}\right)$ is FHP-paracompact if and only if every $\tau_{i}$ open cover of $X$ has a $\tau_{j}$ open $\tau_{j}$ locally finite refinement $(i, j=1,2 ; i \neq j)$.

It is clear that there is no need to restrict the refinement to be a subset of $\tau$. Indeed one can take the members of the refinement from any collection of subsets of $X$ that covers $X$, or better still from a different topology, a third topology on $X$.

For a b.t.s. $\left(X, \tau_{1}, \tau_{2}\right)$ we denote by $L$ the lattice of topologies on $X$ indicated in Figure 1.

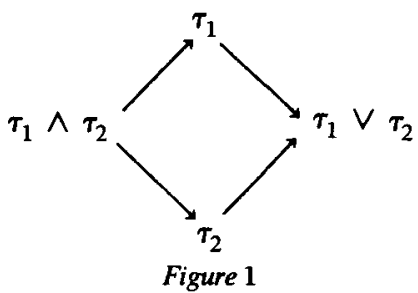

2.1. Definition. Let $\left(X, \tau_{1}, \tau_{2}\right)$ be a b.t.s. Then $X$ is called $(\tau-\sigma)$ paracompact with respect to $\rho$ if and only if every $\tau$ open cover has a $\sigma$ open refinement which is $\rho$ locally finite, where $\tau, \sigma, \rho \in L$.

In particular, $X$ is " $\tau_{1}$ paracompact with respect to $\tau_{2}$ ", if, in Definition 2.1, the choice is $(\tau, \sigma, \rho) \equiv\left(\tau_{1}, \tau_{1}, \tau_{2}\right)$, and $X$ is $R R$-pairwise paracompact if the 
choices are $(\tau, \sigma, \rho) \equiv\left(\tau_{1}, \tau_{1}, \tau_{2}\right)$ and $(\tau, \sigma, \rho) \equiv\left(\tau_{2}, \tau_{2}, \tau_{1}\right)$. The choice in the definition of $F H P$-pairwise paracompactness is $(\tau, \sigma, \rho) \equiv\left(\tau_{i}, \tau_{j}, \tau_{j}\right),(i, j=1,2$; $i \neq j$ ).

2.2. Theorem. Let $\left(X, \tau_{1}, \tau_{2}\right)$ be a b.t.s. Let $\tau, \tau^{\prime}, \sigma, \sigma^{\prime}, \rho, \rho^{\prime} \in$ L. Suppose $X$ is $(\tau-\sigma)$ paracompact with respect to $\rho$. Then $X$ is $\left(\tau^{\prime}-\sigma^{\prime}\right)$ paracompact with respect to $\rho^{\prime}$ if $\tau^{\prime} \leqslant \tau, \sigma \leqslant \sigma^{\prime}$ and $\rho \leqslant \rho^{\prime}$.

The following result is a consequence of [3, Theorem 9].

2.3. TheOREM. Let $\left(X, \tau_{1}, \tau_{2}\right)$ be pairwise Hausdorff. Suppose $X$ is $(\tau-\sigma)$ paracompact with respect to $\rho$. Then $\tau_{1} \subset \tau_{2}$ if $\tau_{1} \leqslant \tau \leqslant \tau_{1} \vee \tau_{2}$ and $\tau_{1} \wedge \tau_{2} \leqslant \sigma$, $\rho \leqslant \tau_{2}$.

The next result is a consequence of [6, Proposition 4].

2.4. TheOREM. Let $\left(X, \tau_{1}, \tau_{2}\right)$ be pairwise Hausdorff. Suppose $X$ is $(\tau-\sigma)$ paracompact with respect to $\rho$. Themn $\tau_{1} \subset \tau_{2}$ if $\tau_{1} \wedge \tau_{2} \leqslant \sigma \leqslant \tau_{1} \leqslant \tau \leqslant \tau_{1} \vee \tau_{2}$ and $\tau_{1} \wedge \tau_{2} \leqslant \rho \leqslant \tau_{2}$

Recall [7, Definition] that $\left(X, \tau_{1}, \tau_{2}\right)$ is $\tau_{1} R \tau_{2}$ if for each $x \in X, \tau_{1} \mathrm{cl}\{x\}=$ $\bigcap\left\{U \mid U \in \tau_{2}\langle x\rangle\right\}=\bigcap\left\{\tau_{1} \operatorname{cl} U \mid U \in \tau_{2}\langle x\rangle\right\}$. Notice in particular that a pairwise Hausdorff space is $\tau_{1} R \tau_{2}$. Recall that a topological space $(X, \tau)$ is called $R_{0}$ if $x \in U \in \tau$ implies $\operatorname{cl}\{x\} \subset U$.

An immediate consequence of [7, Proposition 2] is the following.

2.5. THEOREM. If $\left(X, \tau_{1}, \tau_{2}\right)$ is such that $\left(X, \tau_{1}\right)$ is $R_{0}$, and if $\left(X, \tau_{1}, \tau_{2}\right)$ is $\tau_{1} R \tau_{2}$ and $(\tau-\sigma)$ paracompact with respect to $\rho$, then $\tau_{1} \subset \tau_{2}$ if $\tau_{1} \wedge \tau_{2} \leqslant \sigma \leqslant \tau_{1} \leqslant \tau$ $\leqslant \tau_{1} \vee \tau_{2}$ and $\tau_{1} \wedge \tau_{2} \leqslant \rho \leqslant \tau_{2}$.

\section{Paracompactness in bitopological spaces}

From the remarks in Section 2, we are naturally led to consider the following situations for the property " $(\tau-\sigma)$ paracompact with respect to $\rho$ " in Definition 2.1.
Case (i). $\quad \tau=\tau_{i}, \sigma=\tau_{1} \vee \tau_{2}, \tau=\tau_{j}$
$(i, j=1,2 ; i \neq j)$.
Case (ii). $\quad \tau=\tau_{i}, \sigma=\tau_{1} \vee \tau_{2}, \rho=\rho_{i}$
$(i=1,2)$.
Case (iii). $\quad \tau=\rho_{i}, \sigma=\tau_{i}, \rho=\tau_{1} \vee \tau_{2}$
$(i=1,2)$.
Case (iv). $\tau=\tau_{i}, \sigma=\tau_{1} \vee \tau_{2}, \rho=\tau_{1} \vee \tau_{2} \quad(i=1,2)$. 
3.1. Definition. Let $\left(X, \tau_{1}, \tau_{2}\right)$ be a b.t.s. Let $X$ be $(\tau-\sigma)$ paracompact with respect to $\rho$. Then

in case (i) $X$ is called $\alpha$-pairwise paracompact,

in case (i) $X$ is called $\beta$-pairwise paracompact,

in case (iii) $X$ is called $\gamma$-pairwise paracompact, and

in case (iv) $X$ is called $\delta$-pairwise paracompact.

Note that either of $R R$-pairwise paracompactness or $F H P$-pairwise paracompactness implies $\alpha$-pairwise paracompactness. Also each of $\alpha, \beta$ and $\gamma$-pairwise paracompactness implies $\delta$-pairwise paracompactness.

3.2. TheOREM. Let $\left(X, \tau_{1}, \tau_{2}\right)$ be a b.t.s. Let $\left(X, \tau_{1}\right)$ and $\left(X, \tau_{2}\right)$ be a pair of regular spaces. Then $X$ is $\beta$-pairwise paracompact if and only if $\left(X, \tau_{1}\right)$ and $\left(X, \tau_{2}\right)$ are paracompact.

Proof. This follows from Michael [2, Theorem 2.3(3), page 163].

3.3. THEOREM. Let $\left(X, \tau_{1}, \tau_{2}\right)$ be pairwise Hausdorff and $\alpha$-pairwise paracompact. Then $\tau=\tau_{2}$.

Proof. Let $U$ be a $\tau_{1}$ open set and let $x \in U$. For each $y \in X-U$, there exists a $\tau_{1}$ open $\operatorname{nbd} V_{y}$ of $y$ such that $x \notin \tau_{2} \operatorname{cl} V_{y}$, since the space is pairwise Hausdorff. Then $\mathscr{C}=\left\{V_{y} \mid y \in X-U\right\} \cup\{U\}$ is a $\tau_{1}$ open cover of $X$, so that it has $\tau_{1} \vee \tau_{2}$ open refinement $\mathscr{D}=\left\{W_{y} \mid y \in X-U\right\} \cup\{G\}$. (Such a 'precise' refinement exists.) Further, $\mathscr{D}$ is $\tau_{2}$ locally finite. Let $W=\bigcup\left\{W_{y} \mid y \in X-U\right\}$. Then $\quad X-U \subset \cup\left\{\tau_{2} \operatorname{cl} W_{y} \mid y \in X-U\right\}=\tau_{2} \operatorname{cl} W \subset \bigcup\left\{\tau_{2} \operatorname{cl} V_{y} \mid y \in X-U\right\}$, so that $x \in X-\tau_{2} \mathrm{cl} W \subset U$, where $X-\tau_{2} \mathrm{cl} W$ is $\tau_{2}$ open. Thus $\tau_{1} \subset \tau_{2}$. Reversing the role of $\tau_{1}$ and $\tau_{2}$ in the proof, we get $\tau_{1} \subset \tau_{2}$, so that $\tau_{1}=\tau_{2}$.

3.4. TheOREM. Let $\left(X, \tau_{1}, \tau_{2}\right)$ be pairwise Hausdorff. Let $X$ be $(\tau-\sigma)$ paracompact with respect to $\rho$. Then $\tau_{1} \subset \tau_{2}$ if $\tau_{1} \leqslant \tau \leqslant \tau_{1} \vee \tau_{2}, \sigma \in L$ and $\tau_{1} \wedge \tau_{2} \leqslant \rho$ $\leqslant \tau_{2}$.

Suppose we define $\left(X, \tau_{1}, \tau_{2}\right)$ to be $\tau_{1} \alpha$-paracompact with respect to $\tau_{2}$ if every $\tau_{1}$-open cover admits a $\tau_{1} \vee \tau_{2}$ open refinement which is $\tau_{2}$ locally finite. Then a modification of the proof of Theorem 3.3 leads to the following result, the proof of which is indicated briefly.

3.5. ThEorem. If $\left(X, \tau_{1}, \tau_{2}\right)$ is such that $\left(X, \tau_{1}\right)$ is $R_{0}$, and if $\left(X, \tau_{1}, \tau_{2}\right)$ is $\tau_{1} R \tau_{2}$ and $\tau_{1} \alpha$-paracompact with respect to $\tau_{2}$, then $\tau_{1} \subset \tau_{2}$. 
Proof. Let $V$ be a $\tau_{1}$ open set and let $x \in V$. Then $\tau_{1} \operatorname{cl}\{x\} \subset V$. Also $\tau_{1} \operatorname{cl}\{x\}=\bigcap\left\{U \mid U \in \tau_{2}\langle x\rangle\right\}=\bigcap\left\{\tau_{1} \operatorname{cl} U \mid U \in \tau_{2}\langle x\rangle\right\}$. Then $\mathscr{C}=\left\{X-\tau_{1} \operatorname{cl} U \mid U\right.$ $\left.\in \tau_{1}\langle x\rangle\right\} \cup\{V\}$ is a $\tau_{1}$ open cover of $X$. Proceeding as in Theorem 3.3, with suitable modifications, we arrive at a $\tau_{2}$ open nbd $Q$ of $x$ such that $x \in Q \in V$, whence $\tau_{1} \subset \tau_{2}$.

A consequence of this result is the following result.

3.6. THEOREM. If $\left(X, \tau_{1}, \tau_{2}\right)$ is such that $\left(X, \tau_{1}\right)$ is $R_{0}$, and if $\left(X, \tau_{1}, \tau_{2}\right)$ is $\tau_{1} R \tau_{2}$ and $(\tau-\sigma)$ paracompact with respect to $\rho$, then $\tau_{1} \subset \tau_{2}$ if $\tau_{1} \leqslant \tau \leqslant \tau_{1} \vee \tau_{2}$, $\sigma \in L$ and $\tau_{1} \wedge \tau_{2} \leqslant \rho \leqslant \tau_{2}$.

An immediate consequence of Definition 3.1 (iv) and of the fact that every metrizable space is paracompact is the following result.

3.7. THEOREM. If $\left(X, \tau_{1}, \tau_{2}\right)$ is such that $\tau_{1}$ is induced by a quasimetric $p$ on $X$ and $\tau_{2}$ by its conjugate $q$ on $X$ (i.e., $\tau_{1}=\tau_{p}$ and $\left.\tau_{2}=\tau_{q}\right)$, then $\left(X, \tau_{1}, \tau_{2}\right)$ is $\delta$-pairwise paracompact.

3.8. Remarks. By Theorem 3.7, it is clear that there exists a pairwise Hausdorff $\delta$-pairwise paracompact b.t.s. $\left(X, \tau_{1}, \tau_{2}\right)$ with $\tau_{1} \neq \tau_{2}$. Indeed, such spaces are not $\alpha$-pairwise paracompact.

If $\mathscr{R}$ is the set of real numbers, and if $p$ is the quasimetric defined by $p(x, y)=\min \{1,|x-y|\}$ if $x \leqslant y$ and $p(x, y)=1$ if $y<x$, then we get a quasimetric space $\left(R, \tau_{q}, \tau_{q}\right)$ such that $\tau_{p}$ and $\tau_{q}$ are regular, and such that $\left(R, \tau_{p}\right)$ and $\left(R, \tau_{q}\right)$ are paracompact. Thus, by Theorem $3.2,\left(R, \tau_{p}, \tau_{q}\right)$ is $\beta$-pairwise paracompact. It should be noted that neither $\tau_{p}$ nor $\tau_{q}$ is metrizable.

It has been proved by Stoltenberg [9, Corollary 4.3] that every sequentially compact Hausdorff quasimetric space is metrizable, and by Sion and Zelmar [8, Theorem 2.5] that every compact regular quasimetric space is metrizable. If we consider Helly's space $H$ [4, Example M, page 164], we see that it is compact, Hausdorff, first countable (and hence sequentially compact) and separable; but then it is not metrizable, so that it is not quasimetrizable. It we take this topology to be $\tau_{1}$ and the discrete topology on $H$ to be $\tau_{2}$, then $\left(H, \tau_{1}, \tau_{2}\right)$ is a $\beta$-pairwise paracompact space, and hence $\delta$-pairwise paracompact. However, $\left(H, \tau_{1}, \tau_{2}\right)$ is not quasimetrizable.

There is an example given by Dieudonne of a locally compact Hausdorff space which is not normal. This space is quasimetrizable, as observed by Stoltenberg [9, Example 4.6]. Let us describe this example briefly. Let $X=\left\{\left(m / n^{2}, 1 / n\right) \mid m\right.$ is an integer; $n$ a positive integer $\} \cup\{(x, 0) \mid x \in \mathbb{R}\}$. Let us define a topology $\tau$ on 
$X$ as follows: if $(x, y) \in X$ and $y \neq 0$, define $U_{n}(x, y)=\{(x, y)\}$ and $U(x, 0)=$ $\left\{(u, v) \mid u \leqslant 1 / 2^{n}\right.$ and $\left.|x-u| \leqslant v\right\}$ for each positive integer $n$. Then $\left\{U_{n}(z)\right\}$ forms a nbd base for each $z \in X$ in the topology $\tau$. Thus we get a locally compact Hausdorff but non-normal topological space $(X, \tau)$. Let $U_{0}(x, y)=X$. Define $p$ : $X \times X \rightarrow R$ by $p\left(z, z^{\prime}\right)=1 / 2^{n}$ when $z \in U_{n}\left(z^{\prime}\right)-U_{n+1}\left(z^{\prime}\right)$, where $z, z^{\prime} \in X$. Then $p$ is a quasimetric on $X$ such that $\tau=\tau_{p}$. The topology $\tau_{q}$ induced by the conjugate $q$ is discrete. Thus we see that $\left(X, \tau_{p}, \tau_{q}\right)$ is an example of $\delta$-pairwise paracompact space which is not $\beta$-pairwise paracompact.

It is also clear that if $\left(X, \tau_{1}\right)$ and $\left(X, \tau_{2}\right)$ are regular, then $\left(X, \tau_{1}, \tau_{2}\right)$ is $\gamma$-pairwise paracompact (and hence $\delta$-pairwise paracompact), provided it is $\beta$-pairwise paracompact. However, any space $X$ which has a paracompact topology for $\tau_{2}$ and a non-paracompact one for $\tau_{1}$, with $\tau_{1} \subset \tau_{2}$, is an example of a $\delta$-pairwise paracompact space which is not $\beta$-pairwise paracompact. In particular, if $X=[0, \Omega)$, and if $\tau_{1}$ is the order topology on $X$ and $\tau_{2}$ the discrete topology, then $\left(X, \tau_{1}, \tau_{2}\right)$ is an example of a pairwise Hausdorff biregular, (i.e., $\tau_{1}$ and $\tau_{2}$ are regular) $\delta$-pairwise paracompact space which is not $\beta$-pairwise paracompact. It is to be noted that $\left(X, \tau_{1}, \tau_{2}\right)$ is not quasimetrizable.

We now provide the promised analogue of Michael's theorem for $\delta$-pairwise paracompact spaces which are pairwise regular.

3.9. TheOREM. Let $\left(X, \tau_{1}, \tau_{2}\right)$ be pairwise regular. Then the following are equivalent $(i=1,2)$.

(i) $X$ is $\delta$-pairwise paracompact.

(ii) Every $\tau_{i}$ open cover has $a \tau_{1} \vee \tau_{2}$ open refinement that can be decomposed into an at most countable collection of families of $\tau_{1} \vee \tau_{2}$ open sets which are $\tau_{1} \vee \tau_{2}$ locally finite.

(iii) Every $\tau_{i}$ open cover has a $\tau_{1} \vee \tau_{2}$ locally finite refinement whose members need neither be $\tau_{1} \vee \tau_{2}$ open nor $\tau_{1} \vee \tau_{2}$ closed.

(iv) Every $\tau_{i}$ open cover has a $\tau_{1} \vee \tau_{2}$ locally finite refinement whose members are $\tau_{1} \vee \tau_{2}$ closed subsets of $X$.

Proof. That (i) $\Rightarrow$ (ii) is obvious.

(ii) $\Rightarrow$ (iii). Let $\mathscr{Q}=\left\{U_{\alpha} \mid \alpha \in \Gamma\right\}$ be any $\tau_{i}$ open cover of $X$. There exists, by hypothesis in (ii), a $\tau_{1} \vee \tau_{2}$ open refinement $\mathscr{V}=\left\{\mathscr{V}_{n} \mid n \in Z^{+}\right\}$, where each $\mathscr{V}_{n}=\left\{V_{n \beta} \mid \beta \in \Delta_{n}\right\}$ is a $\tau_{1} \vee \tau_{2}$ locally finite family (but need not be a cover). For fixed $n$, let us write $W_{n}=\bigcup\left\{V_{n \beta} \mid \beta \in \Delta_{n}\right\}$. Then $\left\{W_{n} \mid n \in Z^{+}\right\}$is a $\tau_{1} \vee \tau_{2}$ open cover of $X$. Let $S_{i}=W_{i}-\cup_{j=1}^{i-1} W_{j}$. Then $\left\{S_{n} \mid n \in Z^{+}\right\}$is a cover which is $\tau_{1} \vee \tau_{2}$ locally finite. Thus $\left\{T_{n \alpha}=S_{n} \cap V_{n \alpha} \mid(n, \alpha) \in Z^{+} \times \Gamma\right\}$ is a refinement of $\mathscr{U}$, and it is $\tau_{1} \vee \tau_{2}$ locally finite.

(iii) $\Rightarrow$ (iv). Let $\mathscr{U}=\left\{U_{\alpha} \mid \alpha \in \Gamma\right\}$ be a $\tau_{i}$ open cover of $X$. For each $x$ there exists a $\tau_{i}$ open nbd $U_{x}$ of $x$ with $U_{x} \in \mathscr{U}$. Since $X$ is pairwise regular, there exists 
a $\tau_{i}$ open nbd $V_{x}$ of $x$ such that $x \in V_{x} \subset \tau_{j} \mathrm{cl} V_{x} \subset U_{x}$. Indeed, $x \in V_{x} \subset$ $\left(\tau_{1} \vee \tau_{2}\right) \operatorname{cl} V_{x} \subset \tau_{j} \operatorname{cl} V_{x} \subset U_{x}$. Now $\left\{V_{x} \mid x \in X\right\}$ is a $\tau_{i}$ open cover of $X$. Hence it has a "precise" refinement $\left\{A_{x} \mid x \in X\right\}$ which is $\tau_{1} \vee \tau_{2}$ locally finite, by the hypothesis in (iii). Hence $\left\{\left(\tau_{1} \vee \tau_{2}\right) \mathrm{cl} A_{x} \mid x \in X\right\}$ is a refinement of $\mathscr{U}$ (since $\left.\left(\tau_{1} \vee \tau_{2}\right) \operatorname{cl} A_{x} \subset\left(\tau_{1} \vee \tau_{2}\right) \operatorname{cl} V_{x} \subset U_{x}\right)$ which is also $\tau_{1} \vee \tau_{2}$ locally finite.

(iv) $\Rightarrow$ (i). Let $\mathscr{U}=\left\{U_{\alpha} \mid \alpha \in \Gamma\right\}$ be a $\tau_{i}$ open cover of $X$. Let $\mathscr{V}$ be a $\tau_{1} \vee \tau_{2}$ closed $\tau_{1} \vee \tau_{2}$ locally finite refinement of $\mathscr{U}$. Then each point $x \in X$ has a $\tau_{1} \vee \tau_{2}$ open nbd $W_{x}$ which meets only finitely many members of $\mathscr{V}$. Also, since the space is pairwise regular, there exist $\tau_{i}$ open sets $A_{i}$ and $B_{i}(i=1,2)$ such that $x \in B_{1} \cap B_{2} \subset \tau_{2} \operatorname{cl} B_{1} \cap \tau_{1} \operatorname{cl} B_{2} \subset A_{1} \cap A_{2}=W_{x}$. Since $\left(\tau_{1} \vee \tau_{2}\right) \operatorname{cl}\left(B_{1} \cap\right.$ $\left.B_{2}\right) \subset W_{x}$, if we set $\left(\tau_{1} \vee \tau_{2}\right) \operatorname{cl}\left(B_{1} \cap B_{2}\right)=P_{x}$, then $\left\{P_{x} \mid x \in X\right\}$ is a $\tau_{1} \vee \tau_{2}$ closed covering of $X$ such that each $P_{x}$ meets only finitely many members of $\mathscr{V}$. Then, by [2, Theorem 1.5, page 162], there exists, for each $V \in \mathscr{V}$, an enlargement $E(V)$ in $\tau_{1} \vee \tau_{2}$ and, since $\mathscr{V}$ is a refinement of $\mathscr{U}$, a $U(V)$ in $\mathscr{U}$ such that $V \subset E(V) \cap U(V)$, such that $E(V) \cap U(V)$ is $\tau_{1} \vee \tau_{2}$ open, and such that $\{E(V) \mid V \in \mathscr{V}\}$ is $\tau_{1} \vee \tau_{2}$ locally finite. Therefore $\mathscr{G}=\{E(V) \cap U(V) \mid V \in \mathscr{V}\}$ is a $\tau_{1} \vee \tau_{2}$ open $\tau_{1} \vee \tau_{2}$ locally finite refinement of $\mathscr{U}$.

\section{References}

[1] I. E. Cooke and I. L. Reilly, 'On bitopological compactness', J. London Math. Soc. (2) 13 (1975), 518-522.

[2] J. Dugundji, Topology (Allyn and Bacon, Boston, Mass., 1966).

[3] P. Fletcher, H. B. Hoyle III and C. W. Patty, 'The comparison of topologies', Duke Math. J. 36 (1969), 325-332.

[4] J. L. Kelley, General topology (Van Nostrand, New Jersey, 1955).

[5] J. C. Kelly, 'Bitopological spaces', Proc. London Math. Soc. 13 (1963), 71-89.

[6] T. G. Raghavan and I. L. Reilly, 'Metrizability of quasimetric spaces', J. London Math. Soc. (2) 15 (1977), 169-172.

[7] T. G. Raghavan and I. L. Reilly, 'On nonsymmetric topological structures' (Topology (Proc. Fourth Colloq., Budapest, 1978) Vol. II, Colloq. Math. Soc. Janos Bolyai, Vol. 23, North-Holland, Amsterdam, 1980, pp. 1005-1014).

[8] M. Sion and G. Zelmer, 'On quasimetrizability', Canad. J. Math. 19 (1967), 1243-1249.

[9] R. A. Stoltenberg, 'On quasimetric spaces', Duke Math. J. 36 (1969), 65-71.

[10] W. A. Wilson, 'On quasimetric spaces', Amer. J. Math. 53 (1931), 675-684.

\section{Department of Mathematics}

University of Auckland

Auckland

New Zealand 